\title{
Diagnóstico e intervenção clínica comportamental infantil: uma breve revisão
}

\author{
Maria Cristina O. S. Miyazaki ${ }^{1}$ \\ Faculdade de Medicina S.J.Rio Preto, FAMERP
}

Edwiges F.M. Silvares ${ }^{2}$

Universidade de São Paulo

\begin{abstract}
Alguns transtornos da infância e da adolescência são discutidos, sob um enfoque comportamental, e com a utilização de critérios para diagnóstico do DSM-IV. São fornecidos dados da literatura acerca da manifestação, prevalência, etiologia e prognóstico dos seguintes transtornos: Autismo, Transtorno de Déficit de Atenção/Hiperatividade, Transtornos do Humor (Depressão Maior e Distimia), da Ansiedade (Fobia Especifica e Transtorno de Ansiedade de Separação) e de Expulsão (Enurese e Encoprese). Estratégias de intervenção dentro do enfoque comportamental são também discutidas.

Palavras-chave: terapia comportamental; transtornos da infância e da adolescência; infância e adolescência.
\end{abstract}

\begin{abstract}
Assessment and behavioral clinic intervention with children: a brief review

Some childhood and adolescence disorders are discussed under a behavioral approach, and using DSM-IV diagnosis criteria. Provides data from literature on diagnostic features, prevalence, etiology, and prognosis for the following disorders: autistic disorder, attentiondeficit/hyperactivity disorder, mood disorders (dysthymic disorder and major depressive disorder), anxiety disorders (specific phobia and separation anxiety disorder), and elimination disorders (enuresis and encopresis). Intervention strategies under a behavioral approach are also discussed. Key words: behavior therapy; childhood and adolescence disorders; children and adolescents.
\end{abstract}

A terapia comportamental infantil desenvolveu-se consideravelmente nos últimos anos, caracterizando-se como uma das poucas abordagens que submete seus procedimentos clínicos à avaliação sistemática através de pesquisas científicas (Kazdin, 1991; Kendall \& Morris, 1991; Kendall, 1995).

Avaliação e intervenção têm estreita relação no âmbito da terapia comportamental e algumas questões, propostas por Mash (1989),

1. Chefe do serviço de Psicologia da Faculdade de Medicina de S. J. do Rio Preto. Endereço para correspondência: Coronel Spinola de Castro, 3420 apto 81, Centro, CEP 15015-500, São José do Rio Preto, SP.

2. Professora Doutora do Departamento de Psicologia Clínica da USP. Endereço para correspondência: Rua José Espiridião, 602, CEP 05532-050, São Paulo, SP. devem ser respondidas dentro do processo de decisão envolvido no encaminhamento de uma criança para terapia: Será que essa criança necessita de tratamento? Qual o prognóstico na ausência de intervenção? Que tipo de tratamento tem maiores chances de eficácia, sob responsabilidade de que profissional? Que intervenção tem maiores chances de aceitação por parte da família, da criança e dos membros da comunidade? Quando se deve dar o início e o término da intervenção? Será que a intervenção está tendo o impacto desejado?

Respondidas com o objetivo de fornecer soluções eficazes para os problemas da criança e dos familiares, estas questões têm em vista seu ajustamento a longo prazo. Programas de intervenção em terapia comportamental têm sido 
norteados por dados advindos de desenvolvimentos na área, como compreensão funcional do problema (O'Neill et al., 1990; Silvares, 1991), utilização de uma perspectiva sistêmica (Emery et al., 1992; Fauber \& Long, 1991; Kazdin, 1994; Silvares, 1989; 1991; 1995), considerações acerca do estágio de desenvolvimento do paciente (Rutter, 1986; Peterson et al., 1989), impacto de diferenças individuais, inclusive aspectos biológicos, nos resultados do tratamento (Russo \& Bud, 1987), utilização de diagnósticos clínicos e sistemas classificatórios como auxiliares no processo de decisão de tratamento (Silvares, 1991; Trad, 1989), ênfase em prevenção( Silvares, 1995 ), proliferação de procedimentos comportamentais em instituições de saúde (Amaral \& Yohida, 1993; Domingos, 1993; Miyazaki \& Amaral, 1995; Silvares \& Miyazaki,1994; Tarnowski \& Brown, 1995) e uma crescente aceitação de que tratamentos individualizados devem ser delineados também para populações específicas, isto é, enfocando aspectos relevantes de determinados transtornos da infância e adolescência ( Mash, 1989; Kendall, 1995).Exemplificando o atual estado de conhecimentos na área de terapia comportamental infantil, alguns transtornos serão revistos a seguir.

\section{Autismo}

Crianças autistas apresentam como características principais um acentuado déficit ou inadequação para interações sociais e comunicação, bem como um repertório excessivamente restrito de interesses e atividades. De acordo com o DSM-IV (APA, 1994), o autismo envolve prejuízo qualitativo na interação social e na comunicação, bem como interesses, atividades e padrões comportamentais restritos e estereotipados. Atualmente, a maioria dos autores considera autismo um transtorno do desenvolvimento determinado por fatores biológicos ( Newson \& Rincover, 1989; Rimland, 1991; Schwarztman, 1993) e estudos epidemiológicos sugerem que existem dois a cinco casos de autismo para cada 10000 indivíduos (APA, 1994).

Pesquisas indicam que crianças com esse diagnóstico podem ser divididas em dois grandes grupos, com base em seu rendimento intelectual. O primeiro é composto por crianças com retardo mental severo e profundo, com prognóstico, portanto, reservado, necessitando cuidados básicos por toda a vida. Estratégias de intervenção em relação a esse grupo visam proporcionar o mais alto grau possível de independência (ex.: habilidade de autocuidados, compreensão e obediência a ordens simples, comportamentos sociais básicos, comportamentos de brincar apropriados e redução dos comportamentos autodestrutivos). Um trabalho com os pais é necessário, e envolve treiná-los para ensinar habilidades básicas à criança, controlar comportamentos inadequados e resolver problemas de ordem prática. Além disso, é necessário também ensinar os pais a apreciarem progressos, independente destes serem lentos e em pequeno número, bem como a valorizarem as características únicas e positivas da criança (Johnson \& Werner,1984; Lipp, 1981; Lovaas, 1987). O segundo grupo é constituído por crianças que apresentam nível intelectual normal ou retardo mental leve ou moderado. Para este grupo, além dos objetivos citados para o grupo anterior, há uma ênfase no desenvolvimento de um repertório verbal compatível com faixa etária, interação social com crianças normais, e desenvolvimento de habilidades e comportamentos esperados em crianças pré-escolares e de primeiro grau. Deste grupo, as crianças com melhor prognóstico são aquelas que aprendem rapidamente durante os três ou quatro primeiros meses do tratamento. Os resultados dependem da criança, da qualidade do tratamento e das 
oportunidades disponíveis na vida adulta (Newson \& Rincover,1989). O programa de intervenção deve basear-se em uma avaliação inicial, que inclui descrição operacional dos problemas, freqüência, possíveis variáveis de controle e tentativas anteriores de modificação (Johnson \& Werner, 1984; Keefe, Kopel \& Gordon, 1980; O'Neill et al., 1990).

Geralmente, o início do tratamento do autismo envolve duas tarefas fundamentais. Em primeiro lugar, a criança deve aprender certas habilidades básicas, que constituem prérequisitos para comportamentos mais complexos. Essas habilidades de "prontidão" envolvem comportamentos como sentar, atender ordens e olhar para o material de trabalho. Ao mesmo tempo em que aprende esses requisitos, comportamentos que interferem com a aprendizagem, como birras e agressividade, devem ser minimizados. Esses dois objetivos iniciais ocorrem paralelamente e a forma de atingi-los é descrita com detalhes no trabalho de Lovaas (1987) e Newson \& Rincover (1989).

É preciso lembrar que os conhecimentos sobre autismo são ainda insuficientes, o que faz com que a intervenção seja basicamente sintomática, isto é, "cura" para o autismo, no sentido de um funcionamento "normal", até o momento não existe. Cerca de $10 \%$ das crianças têm um prognóstico bom, isto é, adquirem um padrão de funcionamento nas áreas de linguagem e/ou social e escolar muito próximo da normalidade. Outros $20 \%$ evoluem de forma moderada, com progressos nas áreas social e educacional, apesar de significativo comprometimento da linguagem. Cerca de $70 \%$, entretanto, têm prognóstico "pobre", com progressos limitados e graves comprometimentos em todas as áreas (Newson \& Rincover,1989). Assim, apesar da grande quantidade de estudos sobre autismo, muitas pesquisas são ainda necessárias para uma melhor compreensão desse transtorno.

\section{Transtorno de déficit de atenção/ hiperatividade (TDAH)}

Inúmeros termos já foram utilizados para designar crianças que apresentam déficit em concentrar atenção, dificuldade para controlar impulsos e regular o nível de atividade motora de acordo com as demandas ambientais. Atualmente, o DSM-IV (APA,1994) propõe o termo Transtorno de Déficit de Atenção/Hiperatividade para designar crianças que apresentam déficit de atenção e/ou hiperatividade/impulsividade em grau incompatível com seu nível de desenvolvimento, com prejuízo clinicamente significativo do funcionamento social, acadêmico ou ocupacional.

Estima-se que 3\% a 5\% das crianças em idade escolar apresentem TDAH, com maior freqüência para o sexo masculino (4:1 ou 9:1, dependendo da amostra ser clínica ou da população geral) e em parentes de primeiro grau de crianças com TDAH. O diagnóstico é geralmente realizado quando a criança se encontra no início do primeiro grau, com estabilidade dos sintomas até o início da adolescência e redução destes para grande parte dos indivíduos no final da adolescência e início da idade adulta.

Uma avaliação apropriada requer, além dos critérios do DSM-IV(APA,1994), verificação da idade de início, aspectos ligados ao desenvolvimento, ambiente, motivação da família e da criança para realização do tratamento e diagnóstico diferencial (APA,1994; Hoza et al., 1995). Nos primeiros anos de vida pode ser difícil diferenciar sintomas do TDAH daqueles comportamentos habitualmente presentes em crianças pequenas. Além disso, crianças com rendimento intelectual deficitário ou superdotação intelectual podem apresentar sintomas do transtorno em função de um contexto escolar inadequado, e não da presença real do comprometimento. Crianças com Retardo Mental, 
entretanto, podem receber um diagnóstico adicional de TDAH, caso os sintomas apresentados sejam inadequados para sua idade mental. Crianças provenientes de ambientes caóticos ou desorganizados, ou aquelas com comportamento opositor, ou que podem estar reagindo a um estressor ambiental com impulsividade, problemas de concentração ou inquietação psicomotora, devem também ser diferenciadas daquelas com diagnóstico de TDAH. A utilização de determinados medicamentos pode também causar alteração do comportamento e é importante fator a ser considerado. Deve-se descartar ainda a possibilidade de outros transtornos mentais, como transtornos do humor, da ansiedade, ou alterações comportamentais associadas a doenças orgânicas ou abuso de substâncias. Esses dados devem ser obtidos através de várias fontes e em múltiplos contextos, com o emprego de diferentes estratégias, como escalas que avaliam o comportamento infantil (Achembach, 1991), entrevistas estruturadas (Zimmerman, 1994), observação do comportamento da criança e análise de variáveis ambientais, bem como outras estratégias que enfoquem não apenas os sintomas do transtorno, mas também o funcionamento global (acadêmico e social) e o ambiente familiar.

A cooperação de profissionais de diferentes áreas (ex:psicólogo, médico, professor) é necessária e os dados devem ser analisados dentro de conhecimentos da psicologia do desenvolvimento, uma vez que existe grande variabilidade em relação à capacidade, em termos de tempo de atenção concentrada e controle do comportamento impulsivo, nas diferentes faixas etárias (Barkley, 1989; Hoza et al., 1995; Whalen, 1993). Uma das características dessas crianças é a variabilidade do comportamento ou entre ambientes. Quanto mais estruturado o ambiente e quanto maior o número de demandas, mais o comportamento desvia-se do esperado. Em situações pouco estruturadas e com baixo número de demandas, como brincar livremente, é menos possível distinguir essas crianças de seus colegas "normais".

Crianças com TDAH apresentam ainda maior risco para acidentes, problemas médicos, rendimento acadêmico deficitário, baixa autoestima, depressão, rejeição por parte dos colegas e muitas vezes por parte dos familiares e professores ( Barkley, 1989).

Dois tipos de drogas têm sido freqüentemente utilizadas no tratamento do TDAH: estimulantes, como metilfenidato (ritalina) e antidepressivos tricíclicos (Barkley,1989; Handen,1995). Pesquisas em relação aos estimulantes mais utilizados (por ex: Barkley,1977) indicam uma série de limitações nessa estratégia terapêutica: o comportamento não chega à media esperada em termos de desempenho acadêmico, social e comportamental; os efeitos duram enquanto a droga estiver em ação; existem poucas evidências comprovando mudanças no prognóstico a longo prazo (Barkley, 1989; Pelham \& Murphy, apud Handen, 1995). Dadas as limitações e os efeitos colaterais da medicação - $1 \%$ a $2 \%$ das crianças podem desenvolver tiques motores ou vocais (Barkley, 1988, apud Barkley,1989) - , algumas questões devem ser consideradas antes que essa opção terapêutica seja escolhida. Assim, a idade da criança, a gravidade e duração dos sintomas, o risco de acidentes, o sucesso dos tratamentos anteriores, a ausência de tiques ou de Transtorno de Tourette na família, o nível de ansiedade, a ausência de abuso de substância por parte dos responsáveis e a probabilidade de emprego correto da medicação são fatores a serem considerados na opção.

Diversos estudos mostram que terapia e medicação são estratégias complementares (Handen, 1995; Utrecht, 1993). Entretanto, pesquisas sobre terapia comportamental no TDAH 
têm relatado resultados positivos, sem risco de efeitos colaterais, como ocorre com os psicofármacos. As estratégias utilizadas envolvem procedimentos de modificação de comportamento (ex:reforço, punição, custo de resposta, controle de estímulo) no laboratório, treino de pais para manejo de contingências, treino de professores para manejo em sala de aula (Barkley, 1994) e estratégias cognitivocomportamentais (Barkley, 1989; Hinshaw \& Erhardt, 1991; Serrano, 1991; Kendall \& Braswell, 1993). Estas últimas envolvem treino de competência social, para lidar com a raiva, para modificar estilo de atribuição, de autoinstruções e de auto-avaliação (Hinshaw \& Erhardt, 1991). Serrano (1991) relata estudo de caso em avaliação e intervenção comportamental (com duração de 21 dias) de um garoto de oito anos. Os objetivos terapêuticos foram reduzir comportamentos problema que interferiam com a aprendizagem, aumentar períodos de atenção e de realização de tarefas e aumentar a qualidade do rendimento acadêmico, através de reforçamento de comportamentos incompatíveis. Os resultados atestam a eficácia da intervenção, inclusive generalização dos resultados, e a importância da atenção da professora nesse tipo de programa.

Apesar dos resultados promissores sobre a utilização de tratamentos combinados (terapia mais medicação) e tratamentos utilizando apenas terapia comportamental, muitas pesquisas ainda devem ser realizadas sobre os benefícios, a longo prazo, dos tratamentos para crianças com TDAH.

\section{Transtornos do humor (Depressão)}

Dentre a ampla gama de transtornos da infância e adolescência, a depressão tem suscitado crescente interesse pela freqüência com que este diagnóstico é realizado (Matson,
1989). Diversos aspectos facilitaram a expansão dos estudos sobre depressão na infância e adolescência: precisão cada vez maior dos critérios, diagnósticos e instrumentos para avaliar depressão (ex: DSM-IV, APA,1994; CID, Kovacs, 1992), modelos comportamentais e cognitivo-comportamentais, desenvolvidos para compreender e tratar depressão em adultos, utilizados também para compreender e tratar depressão em crianças e adolescentes (Miyazaki, 1993; Wilkes et al., 1994) e psicopatologia do desenvolvimento, novo campo que desencadeou inúmeros estudos na área (Kovacs, 1989). Um transtorno depressivo inclui alterações comportamentais, cognitivas, emocionais e somáticas (Beck, 1967; Matson, 1989). Vários transtornos da infância e da adolescência, entretanto, podem incluir sintomas de depressão ou coexistir com um quadro depressivo (ex: depressão maior, distimia, transtorno de ansiedade de separação, transtorno de ajustamento com humor deprimido e luto sem complicação, Kazdin, 1989).

Estudos sobre prevalência da depressão na infância e adolescência variam em função da população estudada. Lefkowitz \& Tesiny (1985) identificaram depressão grave em 5,2\% de uma amostra de 3000 crianças normais de terceira, quarta e quinta séries. A prevalência, entretanto, parece ser maior em populações clínicas (2\% a 60\%, Kashani et al., 1981) e populações especiais (Miyazaki, 1993). Em adultos, a depressão é mais freqüente no sexo feminino, mas entre crianças e adolescentes essa diferença nem sempre é encontrada (Lefkowitz \& Tesiny, 1985). Os modelos comportamentais de depressão enfatizam o papel da aprendizagem, das conseqüências ambientais e déficits na aquisição de habilidades, nos problemas de interação com o ambiente apresentados pelo indivíduo deprimido. Ferster (1973) propõe uma análise funcional da depressão, 
com redução na freqüência de certos comportamentos (ex: perda de interesse em atividades habituais) e aumento na freqüência de comportamentos de esquiva e fuga de estímulos aversivos (ex: queixas e freqüentes pedidos de ajuda). Além disso, o indivíduo deprimido apresenta ainda baixa freqüência de comportamentos positivamente reforçados (ex.: interação social). Entretanto, a descrição topográfica do repertório comportamental na depressão não é suficiente para diagnóstico e delineamento de programas de intervenção. É preciso considerar fatores cognitivos, isto é, o significado que esse repertório tem para o indivíduo (Beck et al., 1979; Dattilio, 1995; Kendall, 1995; Lazarus, 1968). Considerando esses dois aspectos, estratégias de intervenção para indivíduos deprimidos têm enfatizado aspectos comportamentais e cognitivos, sendo o tratamento de crianças e adolescentes derivado dos conhecimentos sobre tratamento com adultos.

Apesar da literatura sobre pacientes adultos sugerir que o tratamento combinado (medicação e psicoterapia) é muitas vezes vantajoso, mais pesquisas são necessárias sobre o uso de antidepressivos com crianças. Até o momento, a terapia comportamental tem contribuído de forma significativa para o tratamento da depressão na infância e adolescência. Stark et al. (1991) descrevem componentes da terapia com crianças. Partindo de uma postura colaborativa entre terapeuta e paciente, a criança é ativamente envolvida no processo de avaliação e tratamento, adquirindo compreensão de todo o processo e seus fundamentos. Educação afetiva é um dos procedimentos iniciais e envolve a aquisição, através de jogos e brincadeiras, de conhecimentos acerca da relação entre pensamentos, sentimentos e comportamentos. Estratégias de autocontrole (automonitorização, auto-avaliação, auto-reforçamento) constituem o passo seguinte, acompanhadas de procedi- mentos como role-playing, educação, feedback, modelação, reforçamento social, programação de atividades para aumentar os reforços da criança, treino de habilidades sociais, relaxamento e reestruturação cognitiva. Isso não significa que existe uma receita única de tratamento para todas as crianças e adolescentes deprimidos, mas alguns relatos de casos existentes na literatura podem sugerir linhas de atuação (Garcia \& Rodriguez, 1991; Miller, 1994). Uma ênfase exagerada em técnicas, deixando de lado a compreensão individual de cada caso, tem sido criticada (Evans, 1995; Guilhardi, 1987). Assim, uma avaliação dos pontos fortes e déficits do paciente, bem como o conhecimento de estratégias disponíveis e eficazes devem nortear o delineamento de programas de intervenção.

\section{Transtornos de ansiedade (fobia es- pecífica e transtorno de ansiedade de separação)}

Sentimentos de medo fazem parte da gama de experiências vivenciadas pelos indivíduos ao longo da vida e constituem uma resposta adaptativa a muitas situações, como forma de evitar perigo real. A presença de medos é comum entre crianças e adolescentes, mas estes são geralmente transitórios e fazem parte do desenvolvimento normal. Quando um medo é excessivo ou vinculado a estímulo que não é potencialmente perigoso, interferindo com o funcionamento normal do indivíduo, é denominado fobia (Ross,1979; APA,1994). Um tipo específico de fobia, associado à freqüência à escola, denominado fobia escolar, é queixa comum em clínicas e ambulatórios de psicologia. Embora fobia escolar e transtorno de ansiedade de separação tenham algumas semelhanças, um diagnóstico diferencial deve ser realizado, uma vez que fatores desencadean- 
tes e estratégias de intervenção podem diferir de forma significativa (Silvares \& Souza,1995). A fobia escolar é uma fobia específica, cujos critérios para diagnóstico, são especificados pelo DSM-IV (APA,1994). Envolve medo excessivo e persistente de um objeto ou situação, evitação da situação ou intensa ansiedade (choro, birras ou comportamento adesivo em crianças) para suportá-la, reconhecimento que o medo é excessivo ou irracional (nem sempre presente em crianças), e interfere de forma significativa com o funcionamento psicossocial. A fobia escolar é uma fobia específica tipo situacional, isto é, relacionada a uma situação específica: a escola (APA,1994). Estudos epidemiológicos sugerem que os casos de fobia escolar variam entre 0,4 e 0,7 para cada 100 crianças em idade escolar (Ollendick \& Mayer, apud Mendez \& Macia,1991; Kennedy,1965), sem evidências conclusivas sobre a prevalência do problema em termos de sexo, idade, classe social ou outras variáveis como nível intelectual e rendimento acadêmico (Mendez \& Macia,1991).

Kennedy (1965) classifica a fobia escolar em dois tipos: tipo 1, com início agudo nos primeiros anos de vida escolar; e tipo 2, com diversas recusas anteriores para freqüentar a escola, com início gradual e ocorrendo nas séries mais adiantadas. Em termos comportamentais, devese considerar o sistema triplo de respostas envolvido na fobia escolar: sistema motor (ex:comportamento de evitação, negativismo, comportamento diruptivo), sistema fisiológico (ex:sudorese, tensão muscular, dores) e sistema cognitivo (pensamentos negativos em relação à escola, antecipação de conseqüências desfavoráveis, avaliação negativa da própria capacidade). A fobia escolar parece associada à vivência ou à observação de experiências aversivas associadas ao contexto escolar, podendo ser facilitada por estressores escolares, familiares, doenças prolongadas etc. Algumas características dos pais ou da própria criança, como déficit de assertividade, podem também estar implicadas no quadro. As respostas de esquiva em relação à escola são mantidas por reforçamento negativo (ex:evitação da ansiedade) e positivo (ex:atenção dos pais) (Mendez \& Macia,1991). A avaliação é geralmente realizada através de entrevistas (pais, criança, professores), observações e questionários (Ollendick,1983). Além de avaliar as respostas fóbicas da criança, é importante avaliar também seu funcionamento em outras áreas (ex: social) e reações das pessoas importantes ao problema (ex:pais, professores).

A intervenção comportamental geralmente baseia-se nos princípios do condicionamento clássico e/ou operante. A dessensibilização sistemática (Wolpe,1989), ou algumas de suas variantes, tem sido a estratégia mais freqüentemente empregada. Kennedy (1965) relatou o tratamento bem -sucedido de 50 casos através do procedimento de inundação (Michelson,1989). Outros relatos citam dessensibilização sistemática in vivo, reforço positivo, economia de fichas, contrato de contingências, exposição gradual in vivo e modelação (Mendez \& Macia,1991), colocando sempre a volta imediata à escola (exposição à situação temida) como de extrema importância, uma vez que a perda da continuidade das atividades acadêmicas torna cada vez mais difícil o retorno. Esforços devem também ser feitos para fortalecer comportamentos compatíveis com aprendizagem, auxiliando a criança a alcançar as habilidades que deixou de adquirir durante o período em que esteve ausente da escola.

Enquanto a fobia escolar refere-se a uma dificuldade específica em permanecer na escola, o transtorno de ansiedade de separação envolve um medo excessivo e irreal de separar-se das principais figuras de vinculação (geralmente os pais). É um dos transtornos de ansie- 
dade mais comuns na infância e adolescência, com estudos epidemiológicos indicando que cerca de $41 \%$ das crianças preocupam-se com o tema separação e $2 \%$ a $4 \%$ apresentam o transtorno (APA,1994; Bowen et al.,1990).

O transtorno geralmente desenvolve-se após algum estressor (ex: morte de parente ou animal de estimação, mudança de escola, mudança de vizinhança) e pode ter períodos de remissão e exacerbação (APA,1994).

Diversos procedimentos podem ser utilizados para avaliar o transtorno de ansiedade de separação. Um diagnóstico formal com levantamento dos sintomas normalmente é realizado através de entrevistas, havendo inclusive roteiros de entrevista estruturada para avaliar o transtorno (Zimmerman,1994). Observações do comportamento da criança em diferentes contextos, feitos por observador treinado ou pelos pais ou professores, constituem outra importante fonte de dados. Uma vez que a ansiedade é experimentada pela própria criança, treiná-la para monitorizar seus próprios sintomas de ansiedade é uma estratégia útil, mas que só pode ser empregada com crianças mais velhas (BellDolan,1995; Kendall et al.,1991; Last,1993).

O tratamento comportamental do transtorno de ansiedade de separação é baseado na noção de que o problema é desencadeado e mantido por eventos ambientais. Os tratamentos que envolvem modelação enfatizam a questão da aprendizagem vicariante na questão da separação e utilizam a observação de modelos que exibem comportamento adequado diante da questão da separação. A utilização de manejo de contingências assume que o transtorno relaciona-se às próprias experiências da criança com contingências ambientais e o tratamento baseia-se no reforçamento do comportamento "destemido" (ex: dar adeus aos pais que estão saindo, sem birras). Estratégias como dessensibilização sistemática baseiam-se na noção da inibição recíproca, isto é, redução da ansiedade através da aprendizagem de um comportamento incompatível, geralmente relaxamento. Diversos pesquisadores têm sugerido ainda que a ansiedade pode ser tratada sem uso de relaxamento, apenas com técnicas de exposição. Outros sugerem uma exposição gradual, através de uma hierarquia construída pela criança e terapeuta. A utilização de exposição in vivo ou imaginária depende de vários fatores, como idade da criança e controlabilidade dos estímulos aos quais esta será exposta (Barrios \& O'Dell,1989; Bell-Dolan,1995). O tratamento comportamental dos transtornos da ansiedade na criança e no adolescente envolve estratégias comportamentais, com ênfase adicional nos componentes cognitivos do processamento de informações associado às ansiedades particulares do paciente. O objetivo é ensinar a criança a identificar sinais de ansiedade mal-adaptativa, que sirvam como estímulos discriminativos para a utilização das estratégias adequadas de manejo. Esse enfoque é muito útil quando o principal componente da ansiedade é cognitivo (ex: preocupações com a separação) e quando a criança tem um nível de desenvolvimento que permita o manejo de sua própria ansiedade. Estratégias cognitivas incluem treino de autoinstruções, uma avaliação mais realista da situação causadora da ansiedade, autoreforçamento diante da utilização de estratégias adequadas para enfrentar a ansiedade (BellDolan,1995; Kendall et al., 1991; Kendall,1995). A terapia comportamental, sem dúvida, fornece diversas opções para o tratamento do transtorno de ansiedade de separação. O delineamento da intervenção, entretanto, deve considerar características individuais do paciente, bem como aspectos de ordem prática.

Apesar dos estudos sobre ansiedade de separação estarem contribuindo para um crescente acúmulo do conhecimento na área, muitas 
questões relativas à etiologia, avaliação e tratamento do transtorno precisam ainda ser respondidas (Ollendick \& Huntzinger,1990).

\section{Transtornos de expulsão (enurese e encoprese)}

A aquisição do controle das fezes e da urina é um dos problemas relativos à educação infantil que preocupa sobremaneira os pais. Em uma pesquisa realizada em 1974 ,com 200 pais de crianças em idade pré-escolar, questões acerca de estratégias eficazes para o treinamento da toalete encabeçavam a lista das preocupações parentais (Chamberlain, apud Walker et al., 1989). Esse fato é confirmado pelo número de exemplares vendidos (mais de um milhão de cópias) por Azrin \& Fox (1974) de seu livro Toilet training in less than a day. As questões mais freqüentemente colocadas pelos pais referemse a como e quando iniciar o treino, o que fazer se os resultados ficarem aquém das expectativas, efeitos de um treino muito ou pouco rigoroso, iniciado muito cedo ou tarde etc.

Enurese e encoprese constituem um déficit comportamental na aquisição e manutenção do controle,respectivamente da urina e das fezes, em idade em que a maioria das crianças já adquiriu essa habilidade e na ausência de causa orgânica. Esse déficit constitui motivo de sofrimento para a família e para a criança, que pode sofrer discriminações por parte dos colegas, ser impedida de realizar certas atividades (ex: dormir na casa de colegas) e ter um impacto negativo sobre sua auto-estima.

A prevalência da enurese na idade de cinco anos é de $7 \%$ para o sexo masculino e 3\% para o feminino, aos dez anos, $3 \%$ para o sexo masculino e $2 \%$ para o feminino e aos 18 anos, $1 \%$ para o masculino e menos que isso para o feminino. A enurese pode ainda ser classificada como primária (o controle nunca foi adquirido) e secundária (após período de continência), e, de acordo com os subtipos, apenas diurna, apenas noturna e diurna e noturna (APA,1994). A maioria das crianças torna-se continente até a adolescência e uma relação entre problemas emocionais e enurese não está estabelecida. Em uma revisão da literatura, Shaffer (1973, apud Walker et al.,1989) observou que a maioria das crianças enuréticas não apresenta anormalidades psiquiátricas, sendo que aquelas que têm problemas emocionais não apresentam um padrão consistente de transtorno mental. Outros autores sugerem ainda que quando um problema emocional é identificado em crianças enuréticas, este encontra-se geralmente associado a problemas familiares (ex: separação dos pais) e estressores ambientais (ex: nascimento de irmão). Assim, estressores podem causar enurese mas a presença da enurese não significa necessariamente presença de um problema psicológico (Walker et al.,1989). O modelo da aprendizagem social postula que a enurese é um comportamento deficitário, associado a experiências inadequadas de aprendizagem e/ou contingências inapropriadas de reforçamento. Sem dúvida, intervenções derivadas deste modelo teórico têm se mostrado as formas mais eficazes de tratamento. O "treino da cama seca" proposto por Azrin \& Fox (1974) e Azrin et al. (1973), é um programa que inclui prática positiva, reforçamento positivo, treino para controle de retenção da urina, despertar no meio da noite, reforçamento negativo e treino de limpeza. Diversos estudos sugerem que esse método produz resultados tão positivos quanto a utilização do alarme, sem dúvida, o método mais conhecido para o tratamento da enurese (Doleys, 1989).

O alarme para tratamento da enurese consiste em um dispositivo com sensor para urina colocado na cama ou na roupa da criança (Mowrer \& Mowrer,1938). Um alarme de in- 
tensidade suficiente para despertar a criança é disparado quando a urina atinge o dispositivo, associando, assim, início da micção ao despertar. Diversos estudos mostram cerca de $75 \%$ de eficácia com a utilização do aparelho. Recidivas, entretanto, constituem um problema, ocorrendo em cerca de $40 \%$ dos casos e os resultados positivos têm sido associados à motivação, cooperação parental, tipo de alarme utilizado e avaliação adequada do problema (Doleys, 1989; 1993), exigindo do psicólogo constante exercício de competência (Silvares e Souza, 1996). O alarme de urina, de uso internacional inquestionável, vem sendo desenvolvido no Brasil há menos de uma década (Silvares e Souza, 1996), não sendo encontrado ainda em escala comercial, diferentemente dos países estrangeiros onde, há mais de meio século, tem sido acumulado um corpo de conhecimentos científicos e tecnológicos na área. Estudos sobre o tratamento da enurese diurna têm sido menos freqüentes, mas treino de retenção da urina, treino de limpeza, de discriminação para sensação de bexiga repleta e treino sistemático para utilização da toalete têm sido utilizados com sucesso, dependendo do tipo de dificuldades observadas durante a avaliação (Doleys,1989).

A encoprese tem sido menos estudada que a enurese e, de acordo com o DSM-IV (APA,1994), é definida como a "eliminação repetida de fezes (involuntária ou intencional) em locais inadequados (ex: roupas, chão)" (p.105), em idade em que esse controle já deveria ter sido adquirido e na ausência de causa orgânica. Pode ainda ser ou não acompanhada de constipação intestinal e vazamento do material fecal. Na maioria dos casos (80-95\%) a encoprese está associada à constipação intestinal. A maioria dos encopréticos é do sexo masculino e estimase que cerca de $1 \%$ dos meninos com cinco anos de idade apresentem encoprese.
No enfoque comportamental, a encoprese pode ser compreendida dentro do modelo de aprendizagem social. Doleys (1993) distingue a encoprese primária (ou contínua) de secundária (ou descontínua). Na encoprese primária (não houve aquisição anterior do controle), é preciso avaliar a presença de habilidades que constituem pré-requisitos para a aquisição do controle (ex: tirar a roupa, contração adequada da musculatura), possível ausência de reforçamento para o comportamento adequado de utilização da toalete e aprendizagem da discriminação de dicas fisiológicas que sinalizam necessidade de evacuar. Já a encoprese secundária pode ser explicada por processos de esquiva e condicionamento. A dor ou o medo associados à evacuação podem associar-se ao início da encoprese, com o comportamento de retenção reforçado negativamente pela redução ou evitação da dor. Esse comportamento pode persistir mesmo quando há uma dor mais intensa no momento em que a criança defeca. Outra perspectiva sugere a ocorrência de um condicionamento quando a criança é reforçada pelos pais ao apresentar-se suja. Uma análise funcional é necessária para determinar contingências associadas ao problema $\mathrm{e}$ a terapia envolve uma modificação das contingências inadequadas. É importante notar ainda que diferentes conjuntos de contingências podem desencadear e manter a encoprese, tornando essencial uma análise exaustiva das condições atuais de vida da criança, muito mais que uma ênfase na história passada (Doleys, 1989; 1993; Walker et al., 1989).

\section{Conclusão}

Mesmo abordando apenas alguns dos transtornos que podem estar presentes na infância e adolescência e reconhecendo a necessidade de maiores pesquisas, é possível observar, 
com base na revisão da literatura aqui realizada, que a terapia comportamental dispõe de estratégias para lidar satisfatoriamente com estes problemas. Considerar mudanças inerentes ao processo de desenvolvimento, delinear o processo de intervenção levando em conta especificidades do problema apresentado e características individuais do paciente, bem como a integração de procedimentos cognitivos e comportamentais têm favorecido o desenvolvimento da área.

\section{Referências}

Achenbach, T.M.(1991). Manual for the Child Behavior Checklist/4-18 and TRF profile. Burlington, VT:U Vt Dept.Psychiat.

Amaral, V. A.R. \& Yoshida, G.O. (1993). A psicologia como profissão da área da saúde, a prática, a supervisão e a pesquisa: relato de uma experiência. Estudos de Psicologia,10(3): 85-96.

American Psychiatric Association (1994). Diagnostic and Statistical Manual of Mental Disorders DSM-IV. Washington, D.C.:APA.

Azrin, N.H., Sneed, T.J. \& Fox, R.M. (1973). Dry bed: a rapid method of eliminating bedwetting (enuresis) of the retarded. Behoviour Research \& Therapy, 11:427-434.

Azrin, N.H. \& Fox, R.M.(1974). Toilet training in less than a day. New York: Pocket Books.

Barkley, R.A. (1977). A review of stimulant drug research with hyperactive children. Archives of General Psychiatry,36:201-208.

Barkley, R.A.(1989). Attention deficithyperactivity disorder. In: E.J. MASH, E. J. \& R.A. Barkley (eds.) Treatment of Childhood Disorders. New York: Guilford. Pp. 39-72.

Barkley, R. (1994). ADHD in the classroom. Strategies for teachers [vídeo]. New York:Guilford.

Barrios, B.A. \& O'Donell, S.L.(1989). Fears and anxieties. In: E.J. Mash, \& R.A.Barkley (ed.)
Treatment of childhood disorders. New York:Guilford. pp.167-221.

Beck, A.T.(1967). Depression: causes and treatment. Philadelphia:University of Pennsylvania Press.

Beck,A.T., Rush, A.J., Shaw, B.F. \& Emery, G. (1979).Terapia cognitiva da depressão. Trad. Vera Ribeiro. Rio de Janeiro:Zahar.

Bell-Dolan, D. (1995). Separation Anxiety Disorder. In: R.T. Ammerman \& M. Hersen (ed.) Handbook of Child Behavior Therapy in the Psychiatric Setting. New York:Wiley. pp.217-238.

Bowen, R.C., Offord, D.R. \& Boyle, M.H. (1990). The prevalence of overanxious disorder and separation anxiety disorder. Results from the Ontario child health study. Journal of the American Academy of Child and Adolescent Psychiatry, 29:753-758.

Dattilio, F. (1995). Formação em Terapia Cognitiva. Workshop, São Paulo.

Doleys, D.M.(1989). Functional enuresis and encopresis. In: C.G. Last \& M. Hersen (ed.) Handbook of child psychiatric diagnosis. New York:Wiley. pp.427-442.

Doleys, D.M. (1993). Enuresis y encopresis. In: T.H. Ollendick, T.H. \& M. Hersen (ed.) Psicopatologia infantil. Barcelona: Martinez Roca. pp.338-365.

Domingos, N.A.M. (1993). Preparo para cirurgia: teste de programas psicológicos na redução de ansiedade de crianças e mães. Campinas, Dissertação (mestrado). PUCCAMP. 166p.

Emery, R.E., Fincham, F.D. \& Cummings, E.M. (1992). Parenting in context: systemic thinking about parental conflict and its influence on children. Journal of Consulting and Clinical Psychology, 60(6):909-912.

Evans, I.M. (1995). More clinical science, please: hold the technology. Apresentado ao World Congress of Behavioural and Cognitive Therapies, Copenhagen, Dinamarca.

Fauber, R.L. \& Long, N. (1991). Children in context: the role of the family in child psychotherapy. Journal of Consulting and Clinical Psychology, 59 (6): 813-820. 
Ferster, C.B. (1971). A functional analysis of depression. American Psychologist, 23 (10): 857870 .

Garcia, J. \& Rodriguez, J. (1991). Depressión en la adolescencia: presentación de un caso. In: F.X. Mendez \& D.M. Antón (coord.) Modificación de conducta con niños y adolescentes: libro de casos. Madrid:Pirámide. pp.108-128.

Guilhardi, H.J. (1987). A formação do terapeuta comportamental: que formação? In: H.W. Lettner \& B.P. Rangé. Manual de psicoterapia comportamental. São Paulo:Manole. pp.313-320.

Handen, B.L. (1995). Behavior therapy and pharmacological adjuncts. In: R.T. Ammerman \& M. Hersen (ed.) Handbook of Child Behavior Therapy in the Psychiatric Setting. New York:Wiley. pp.109-132.

Hinshaw, S.P. \& Erhardt, D. (1991). Attentiondeficit Hyperactivity Disorder. In: P.C. Kendall (ed.) Child \& Adolescent Therapy: cognitivebehavioral procedures. New York:Guilford. pp.98-128.

Hoza, B., Vallano, G. \& Pelham Jr, W.E. (1995). Attention-Deficit/ Hyperactivity Disorder. In: R.T. Ammerman \& M. Hersen. Handbook of Child Behavior Therapy in the Psychiatric Setting. New York:Wiley. pp.181-198

Johnson, V.M. \& Werner, R.A. (1984). Um guia de aprendizagem progressiva para crianças retardadas. Trad. Ana Fátima R. Alves. São Paulo:Manole.

Kashani, J.H., Husain, A., Shekim, W.O., Hodges, K.H., Cytryn, L. \& Mcknew, D.H. (1981). Current perspectives on childhood depression: an overview. American Journal of Psychiatry, 138: 143-153.

Kazdin, A.E. (1989). Childhood depression. In: E.J. Mash \& R.A Barkley (ed.) Treatment of Childhood Disorders. New York:Guilford. pp.135166.

Kazdin, A.E. (1991).Effectiveness of psychotherapy with children and adolescents. Journal of Consulting and Clinical Psychology, 59 (6): 785798.
Kazdin, A.E. (1994).Adversidad familiar, desvantajas socioeconomicas y estres en los padres: variables contextuales relacionadas con la terminación prematura de la terapia conductual infantil. Psicologia Conductual, 2 (1): 5-21.

Keefe, F.J., Kopel, S.A. \& Gordon, S.B.(1980). Manual prático de avaliação comportamental. Trad. Paulo C. Geraldes. São Paulo:Manole.

Kendall, P.C. (1995). Cognitive Therapy with Children. Workshop, World Congress of Behavioural and Cognitive Therapy, Copenhagen, Dinamarca.

Kendall, P.C. \& Braswell, L. (1993). Cognitivebehavioral therapy for impulsive children. 2nd ed. New York:Guilford.

Kendall, P.C. \& Morris, R.J. (1991). Child Therapy: issues and recomendations. Journal of Consulting and Clinical Psychology,59(6):777-784.

Kendall, P.C., Chansky, T.E., Freidman, M., Kim, R., Kortlander, E., Sessa, F. \& Siqueland, L. (1991). Treating anxiety disorders in children and adolescents. In: P.C. Kendall (ed.) Child and adolescent therapy: cognitive-behovioral procedures. New York:Guilford. pp.131-164.

Kennedy, W.A. (1965). School phobia: rapid treatment of fifty cases. Journal of Abnormal Psychology, 70:285-289.

Kovacs, M. (1989). Affective disorders in children and adolescents. American Psychologist, 44(2):209-215.

Kovacs, M. (1992). Children's Depression Inventory: Manual. New York:MHS.

Last, C.G. (1993). Trastornos por ansiedad. In: T.H. Ollendick \& M. Hersen (ed.) Psicopatologia Infantil. Barcelona:Martinez-Roca. pp.257-266

Lazarus, A.A. (1968). Learning theory and the treatment of depression. Behaviour Research \& Therapy, 6: 83-89.

Lefkowitz, M.M. \& Tesiny, E.P. (1985). Depression in children: prevalence and correlates. Journal of Consulting and Clinical Psychology, 53:647-656

Lipp, M.E.N. (1981). Sexo para deficientes mentais: sexo e excepcional dependente e não- 
dependente. Campinas:Cortez/Autores Associados.

Lovaas, O.I. (1987). Behavioral treatment and normal education and intellectual functioning in young autistic children. Journal of Consulting and Clinical Psychology, 35 (1):3-9.

Mash, E.J. (1989). Treatment of child and family disturbance: a behavioral systems perspective. In: E.J. Mash \& R.A. Barkley (ed.) Treatment of Childhood Disorders. New York:Guilford. pp.336

Matson, J. (1989). Treating depression in children and adolescents. New York: Pergamon.

Mendez, F.X. \& Macia, D. (1991). Tratamiento conductual de un caso de fobia escolar. In: F.X. Mendez \& D.M. Antón (coord.) Modificación de conducta con niños y adolescentes: libro de casos. Madrid:Pirámide. pp.74-107.

Michelson, L. (1989). Flooding. In: A. S. Bellack \& M. Hersen (ed.) Dictionary of Behavior Therapy Techniques. New York:Pergamon. pp.126-131.

Miller, I.W. (1994). Major depression. In: C.G. Last \& M. Hersen (ed.) Adult Behavior Therapy Casebook. New York:Plenum. pp.33-60.

Miyazaki, M.C.O.S. (1993). Enfoque psicossocial da doença crônica: depressão em pacientes pediátricos portadores de asma e suas mães. Campinas, Dissertação (mestrado). 186p.

Miyazaki, M.C.O.S. \& Amaral, V.L.A.R. (1995). Instituições de saúde. In: B. Rangé (org.) Terapia Comportamental e CognitivoComportamental. Pesquisa, Prática, aplicações e problemas. Campinas, Psy II.

Mowrer, O.H. \& Mowrer, W.M. (1938). Enuresis-a method for its study and and treatment. American Journal of Orthopsychiatry, 8:436-459.

Newson, C. \& Rincover, A. (1989). Autism. In: E.J. Mash \& R.S. Terdal (ed.) Treatment of Childhood Disorders. New York:Guilford. pp.286346.

Ollendick, T.H. (1983). Reliability and validity of the Revised Fear Survey Schedule for Children (FSSC-R). Behoviour Research and Therapy, 21:685692.
Ollendick, T.H. \& Huntzinger, R.M.(1990). Separation anxiety disoder in childhood. In: Hersen,M. \& Last,C.G.(ed) Handbook of Child and Adult Psychopathology: a longitudinal perspective. New York, Pergamon. pp.133-149.

O'neill, R.E., Horner, R.H., Albin, R.W., Storey, K. \& Sprague, J.R. (1990). Functional analysis of problem behavior: a practical assessment guide. Sycamore,Il:Sycamore.

Peterson, L., Burbach, D.J. \& Chaney, J. (1989).Developmental issues. In: C.G. Last. \& M. Hersen Handbook of Child Psychiatric Diagnosis. New York:Wiley. pp.463-482.

Rimland, B. (1991). Research on the diagnosis, cause, and treatment of children and adults with autism. Anais do IV Congresso Mundial da Criança Autista. São Paulo.

Ross, A.O. (1979). Distúrbios psicológicos na infância e adolescência. São Paulo: McGrawHill.

Russo, D.C. \& Bud, K.S. (1987). Limitations of operant practice in the study of disease. Behavior modification, 11: 264-285.

Rutter, M. (1986). The developmental psychopathology of depression: issues and perspectives. In: M. Rutter, C.E. Izard \& P.B. Read (ed.) Depression in young people: developmental and clinical perspectives. New York:Guilford. pp.330.

Schwartzman, J.S. (1993). Autismo infantil. Temas sobre Desenvolvimento, 2 (10): 3-28.

Serrano, I. (1991). Tratamento conductual de un niño hiperactivo. In: F.X. Méndez \& D.M. Antón (coord.) Modificación de conducta conniños y adolescentes: libro de casos. Madrid:Pirámide. pp.349-376.

Silvares, E.F.M. (1989). Aconselhamento de pais: uma breve análise retrospectiva de duas tendências comportamentais (Ecologia e Generalização). Boletim de Psicologia, 38 (90-91): 3743.

Silvares, E.F.M. (1991). A evolução do diagnóstico comportamental. Psicologia: Teoria \& Pesquisa, 7 (2): 179-187. 
Silvares, E.F.M. (1993).O papel preventivo das clínicas-escola de psicologia em seu atendimento a crianças. Temas em Psicologia, 2:87-97.

Silvares, E.F.M. (1995). O modelo triádico no contexto da terapia comportamental com famílias. Psicologia: Teoria e Pesquisa ,11(3):235-241.

Silvares, E.F.M. \& Miyazaki, M.C.O.S. (1994). Behavioral assessment of patients undergoing endoscopic examination. Apresentada à 15th International Conference of the Stress and Anxiety Research Society. Madri:Espanha.

Silvares, E.F.M. \& Souza, C.L. (1995). Medo da escola: o médico pode ajudar? Revista de Pediatria Moderna, XXXI (5): 829-833.

Silvares, E.F.M. \& Souza, C.L. (1996). Enurese noturna: um tratamento alternativo. Revista de Pediatria Moderna, XXXII (6):662-667

Stark, K.D., Rouse, L.W. \& Livingston, R. (1991). Treatment of depression during childhood and adolescence: cognitive-behavioral procedures for the individual and family. In: P.C. Kendall (ed) Child and adolescent therapy: cognitive behavioral procedures. New York:Guilford. pp.165-206.

Tarnowski, K.J. \& Brown, R.T. (1995). Pediatric pain. In: R.T. Ammerman, R.T. \& M. Hersen (ed.) Handbook of Child Behavior Therapy in the Psychiatric Setting. New York:Wiley. pp.453476.

Trad, P. (1989). A nosological approach to assessing childhood psychiatric disorders. In: C.G. Last \& M. Hersen (ed.) Handbook of Child Psychiatric Diagnosis. New York:Wiley. pp.12-27.

Utrecht, U. (1993). Pharmacotherapy and behaviour therapy: Competition or cooperation? Acta Paedopsychiatrica: International Journal of Child \& Adolescent Psychiatry, 56 (2): 123-127.

Walker, C.E., Kenning, M. \& Faust-Campanile, J. (1989). Enuresis and encopresis. In: E.J. Mash \& R.A. Barkley (ed.) Treatment of childhood disorders. New York:Guilford. pp.423-448.

Whalen, C.K. (1993). Trastorno por déficit de atención con hiperatividad. In: T.H. Ollendick \& M. Hersen (eds.) Psicopatología Infantil. Madrid:Martinez-Roca. pp.159-202.
Wilkes, T.C.R., Belsher, G., Rush, A.J. \& Frank, E. (1994). Cognitive Therapy for Depressed Adolescents. New York:Guilford.

Wolpe, J. (1989). Systematic desensitization. In: A.S. Bellack \& M. Hersen (ed.) Dictionary of Behavior Therapy Techniques. New York:Pergamon. pp.215-219.

Zimmerman, M. (1994). Interview Guide for Evaluating DSM-IV Psychiatric Disorders and the Mental Status Examination. Philadelphia:Psych Press Products. 